\title{
Neurorehabilitation nach Hirnschlag: Alter ist kein limitierender Faktor*
}

\author{
Joachim W. Kocha, \\ Fabio Baronti ${ }^{b}$, \\ Urs Hürlimann ${ }^{c}$

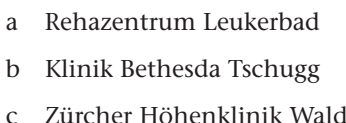 \\ c Zürcher Höhenklinik Wald
}

* Vgl. den Artikel von Beer S, Clarke S, Diserens K, Engelter S, Müri R, Schnider A, Urscheler N. Neurorehabilitation nach Hirnschlag. Schweiz Med Forum 2007;7(12):294-297.

Korrespondenzen:

Dr. med. Joachim W. Koch

Rehazentrum Leukerbad

Neurologie

Postfach

CH-3954 Leukerbad

j.w.koch@rzl.ch

Dr. med. Fabio Baronti

Klinik Bethesda Tschugg

CH-3233 Tschugg

baronti.f@klinik-bethesda.ch

Dr. med. Urs Hürlimann

Winterthur Versicherungen

Medizinischer Dienst

Brandschenkestrasse 24

CH-8027 Zürich

urs.huerlimann@winterthur.ch

\section{Zusammenfassung}

Die Arbeitsgruppe «Neurorehabilitation im Alter» der SNRG hat die vorhandene Erfahrung und Literatur zu diesem Thema evaluiert und kommt zu folgendem Schluss:

- ältere Patienten profitieren von einer spezifischen Neurorehabilitation;

- neurorehabilitative Massnahmen müssen auch bei älteren Patienten so früh wie möglich geplant und eingeleitet werden («time is brain, time is disability, competence is brain»).

Für die klinische Praxis bedeutet dies, dass Patienten den Anspruch auf eine Neurorehabilitation nicht ab einem bestimmten Alter verlieren. Nach einem Hirnschlag muss bei älteren Patienten ebenso wie bei jüngeren Patienten die Indikation neurorehabilitativer Massnahmen geprüft und bei positiver Beurteilung eine stationäre Neurorehabilitation eingeleitet werden.

Das Alter als limitierenden Faktor zu betrachten, widerspricht dem heutigen medizinischen Kenntnisstand. Auch ist es nicht angebracht, bei älteren Patienten eine Neurorehabilitation erst zu einem späteren Zeitpunkt, mehrere Wochen oder Monate nach dem Hirnschlag, einzuleiten, d.h., eine Selektion entsprechend dem Ausmass der Spontanremission vorzunehmen.

Der Bericht wurde an der Mitgliederversammlung der SNRG vom 29. September 2006 diskutiert und in der abgedruckten Form von den Mitgliedern genehmigt.

\section{Evaluation der Literatur}

Der Hirnschlag ist in den industrialisierten Ländern heute die häufigste Ursache für schwere und dauernde Behinderung sowie für bleibende Hilfs- und Pflegebedürftigkeit. Mit der steigenden Lebenserwartung der Bevölkerung ist in den kommenden Jahren auch mit einer Zunahme der Hirnschlaginzidenz und -prävalenz zu rechnen.

Aufgrund seiner Häufigkeit steht der Hirnschlag für die neurologische und neurorehabilitative Therapie im Vordergrund. Seine gesellschaftlichen Auswirkungen (Tod und Behinde-

\section{Neuroréadaptation après un} accident vasculaire cérébral:

\section{l'âge n'est pas un facteur limitant}

Le groupe de travail «Neuroréadaptation des personnes âgées» de la SNRG a évalué les expériences faites ainsi que la littérature à ce sujet et en tire les conclusions suivantes:

- les personnes âgées profitent d'une neuroréadaptation spécifique;

- il est indispensable de planifier et de préparer le plus tôt possible des interventions de réadaptation neurologique chez des personnes âgées («time is brain, time is disability, competence is brain»).

Quant à la pratique clinique, cela signifie qu'à partir d'un certain âge les patients ne perdent pas le droit d'une neuroréadaptation. Après un accident vasculaire cérébral il faut examiner autant chez des patients âgées que chez des jeunes la nécessité d'interventions neurorééducatifs afin de mettre en chemin une réadaptation neurologique stationnaire en cas d'une appréciation positive.

Il est contradictoire de considérer l'âge comme un facteur limitant face aux connaissances médicales actuelles. Aussi n'est-il pas opportun de commencer une réadaptation neurologique chez des personnes âgées à un moment plus tard, plusieurs semaines ou mois après l'accident vasculaire cérébral, c'est à dire d'entreprendre une sélection en mesures des remissions spontanées.

Le rapport à été discuté lors de l'assemblée générale de la Société Suisse de Neurorééducation du 29 septembre 2006 et à été approuvé par les membres en version imprimée. 
rung, Therapie- und Invaliditätskosten) machen ihn zu einem Brennpunkt der aktuellen Gesundheitspolitik.

Die Prognose des Hirnschlags hat sich in den letzten Jahren durch eine Spezialisierung der Akuttherapie und der Rehabilitation wesentlich verbessert. Die Behandlung auf einer spezialisierten Stroke Unit mit unmittelbarer Einleitung kurativer und rehabilitativer Massnahmen in Form einer koordinierten, multiprofessionellen und interdisziplinären Teamarbeit reduziert Mortalität und verbleibende schwere Behinderungen ohne Verlängerung der Dauer des stationären Aufenthalts. Dies konnte in mehreren Studien und grossen Metaanalysen nachgewiesen werden [1-3]. Durch eine Vielzahl von Studien wurde nicht nur die Wirksamkeit rehabilitativer Massnahmen nach Hirnschlag aufgezeigt, sondern auch deren Wirkprinzip in Form einer Förderung der neuronalen Plastizität des Gehirns.

Trotz des nachgewiesenen und heute allgemein akzeptierten Nutzens der Hirnschlagrehabilitation werden Art und Umfang der Massnahmen derzeit vorrangig unter dem Gesichtspunkt der Kosten diskutiert. Insbesondere wird in diesem Zusammenhang neben dem Schweregrad des Hirnschlags häufig das Alter als limitierender Faktor angeführt. So wird bei älteren Patienten nach Hirnschlag der Erfolg von spezifischen Rehabilitationsmassnahmen in Frage gestellt. In der klinischen Praxis werden ältere Patienten auch seltener in eine stationäre Neurorehabilitation überwiesen [4]. Dies steht im Widerspruch zu den erwähnten Studien. Der Therapieerfolg (Outcome) einer spezialisierten Akutbehandlung und Rehabilitation (Stroke Unit) zeigte weder eine Abhängigkeit vom Schweregrad des Hirnschlags noch vom Alter der Patienten [2].

Die vorliegenden Studiendaten, insbesondere die neuere Literatur, die auch die Fortschritte und Spezialisierung der neurorehabilitativen Therapie des Hirnschlags der letzten Jahre berücksichtigt, widersprechen der genannten klinischen Praxis und begründen eine spezifische Neurorehabilitation auch bei älteren Patienten nach Hirnschlag.

- In einer Luzerner Studie [5] zeigte sich während der stationären Neurorehabilitation nach Hirnschlag kein Unterschied bezüglich Verbesserungen der Aktivitäten des täglichen Lebens bei Patienten im Alter von 65 bis 74 Jahren verglichen mit älteren Patienten (75-93 Jahren).

- In einer dänischen Studie (Copenhagen Stroke Study) wurde eine Abhängigkeit des Hirnschlag-Outcomes vom Alter der Patien- ten aufgezeigt. Ältere Patienten zeigten in dieser Studie jedoch bereits initial ein grösseres neurologisches Defizit und eine geringere Selbständigkeit in den Alltagsaktivitäten (Barthel-Index). Im Verlauf war bei älteren Patienten die Verbesserung der Alltagskompetenz geringer als bei jüngeren, hingegen zeigte sich kein Einfluss des Alters auf das Ausmass der neurologischen Verbesserung oder die Geschwindigkeit der Erholung. Von den Autoren wurde empfohlen, bei älteren Patienten den Schwerpunkt der Rehabilitation auf die Verbesserung der Aktivitäten des täglichen Lebens zu legen, das Alter selbst sollte kein Selektionskriterium für die Rehabilitation sein [6].

- Eine neuere italienische Studie [7] zeigte eine Abhängigkeit des Alters auf die Selbständigkeit in den Aktivitäten des täglichen Lebens und deren Verbesserung während der Rehabilitation. Ein positiver Effekt der stationären Rehabilitation wurde jedoch auch bei Patienten über 85 Jahre aufgezeigt.

- In einer griechischen Studie war die Wirksamkeit der stationären Neurorehabilitation bei älteren Patienten hinsichtlich einer funktionellen Verbesserung (FIM) geringer als bei jüngeren, die Mehrheit der älteren Patienten konnte jedoch erfolgreich am Rehabilitationsprogramm teilnehmen und nach Hause zurückkehren [8].

- Für sehr alte Patienten ( $\geq 85$ Jahre) zeigte sich in einer israelischen Studie eine geringere Erfolgsrate der stationären Neurorehabilitation, die Dauer der Rehabilitation und die Komplikationsraten waren aber im Vergleich mit jüngeren Patienten ohne Unterschied. Insgesamt kamen die Autoren zu dem Schluss, dass eine Rehabilitation bei sehr alten Patienten nicht weniger gerechtfertigt erscheint als bei jüngeren [9].

- In einer prospektiven kanadischen Studie [10] wurde der Einfluss des Alters auf die funktionelle Erholung im Rahmen einer stationären Hirnschlagrehabilitation untersucht. Das Alter hatte einen negativen Einfluss auf den funktionellen Status bei Entlassung, gemessen mit dem Gesamt-FIM-Score und dem motorischen FIM-Score, aber nicht in bezug auf die Veränderung der FIM-Scores während der Rehabilitation. Insgesamt erklärte ein höheres Alter nur 1,3\% der Variation im funktionellen Outcome nach Berücksichtigung anderer Faktoren, wie dem FIM-Score bei Eintritt. Die klinische Relevanz dieses geringen Effekts wurde in Frage gestellt, und es wurde als nicht gerechtfertigt erachtet, eine Rehabilitation 
allein aufgrund eines fortgeschrittenen Alters zu verneinen.

- Zu der gleichen Schlussfolgerung kommen die Autoren einer retrospektiven chinesischen Studie mit knapp 900 Patienten [11]. Beim Vergleich dreier Altersgruppen (<65 Jahre; 65-79 Jahre; $\geq 80$ Jahre) ergab sich zwar eine Abhängigkeit des funktionellen Status (Gesamt-FIMScore) zu Beginn und am Ende der Rehabilitation vom Alter der Patienten, das Alter war jedoch kein unabhängiger Prädiktor für ein gutes Outcome. Es zeigten sich keine Unterschiede zwischen den Altersgruppen hinsichtlich einer Verbesserung des FIM-Scores während der Rehabilitation. Ältere Patienten profitierten gleichermassen von einer stationären multidisziplinären Neurorehabilitation.

- Nach den aktuellen Daten der Schlaganfalldatenbank Hessen erhielten nur 13,5\% der Patienten über 85 Jahre im Vergleich zu über $30 \%$ der Patienten zwischen 60 und 69 Jahren eine stationäre Rehabilitation. Das Alter stellt jedoch keinen limitierenden Faktor in der Rehabilitation nach Hirnschlag dar, wie die Auswertung der genannten Schlaganfalldatenbank zeigte. Sowohl in der frühen Phase nach Hirnschlag [12] als auch für die weiterführende Rehabilitation [4] konnte nachgewiesen werden, dass auch ältere Patienten von den Massnahmen profitieren. Die Datenbank erfasste insgesamt 14884 Patienten, die zwischen 1999 und 2003 in einer neurologischen Rehabilitationsklinik behandelt wurden. Ein höheres Alter der behandelten Patienten hatte keinen negativen Einfluss auf den Rehabilitationserfolg, gemessen als tägliche Verbesserung des Barthel-Indexes. Die Verbesserung des Barthel-Indexes pro Tag nahm sogar mit steigendem Alter von 0,34 (Patienten jünger als 55 Jahre) bis auf 0,73 in der Altergruppe über 85 Jahre signifikant zu.

Insgesamt widerlegen die Untersuchungen die Behauptung, ältere Hirnschlagpatienten könnten allgemein nicht von einer spezifischen multidisziplinären Rehabilitation profitieren. Da ältere Patienten häufig bereits bei Beginn der Neurorehabilitation einen schlechteren funktionellen Status aufweisen, ist die alleinige Betrachtung des funktionellen Status bei Austritt unzureichend. Aussagekräftiger sind die funktionellen Verbesserungen während der Neurorehabilitation. Hier zeigen die neueren Untersuchungen von Bagg et al. [10], Luk et al. [11] und Misselwitz et al. [4], dass ein höheres Alter keinen negativen Einfluss auf den Rehabilitationserfolg hat, gemessen am Funktionszuwachs (Verbesse- rung von Barthel-Index, FIM-Score) während der Neurorehabilitation.

Der Anspruch auf eine Neurorehabilitation nach Hirnschlag ist nicht alterslimitiert. Dies gilt grundsätzlich auch für Patienten mit Komorbiditäten. Nur wenn eine Komorbidität eindeutig im Vordergrund steht und auch mit hoher Wahrscheinlichkeit die Teilnahme an einem intensiven multidisziplinären Rehabilitationsprogramm verunmöglichen wird, ist es vertretbar, bei älteren Patienten nach Hirnschlag keine spezifische Neurorehabilitation durchzuführen.

Verschiedene internationale Organisationen weisen in ihren Vernehmlassungen auf die Notwendigkeit von Rehabilitation und Wiedereingliederungsmassnahmen hin.

Die Weltgesundheitsorganisation (WHO) erachtet es als verbindlichen Grundsatz, dass die einzelnen Länder nationale Rehabilitationsprogramme für alle Personengruppen mit Behinderungen (disabilities) entwickeln, basierend auf den Prinzipien einer uneingeschränkten Teilhabe (Partizipation) und Gleichberechtigung [13].

Gemäss den Vereinten Nationen sollen alle Patienten mit Behinderungen, einschliesslich der Personen mit schweren und/oder multiplen Behinderungen, welche einer Rehabilitation bedürfen, auch Zugang zu dieser haben [14].

Mit dem Ziel, die Lebensqualität behinderter Menschen in Europa in den Jahren 2006-2015 zu verbessern, wurde vom Ministerkomitee des Europarats ein Aktionsplan zur Förderung der Rechte und vollen Teilhabe behinderter Menschen an der Gesellschaft aufgestellt [15].

Durch eine geeignete Akuttherapie und Rehabilitation soll laut der 2. Helsingborg Consensus Conference bis zum Jahr 2015 das Ziel erreicht werden, dass in Europa über 70 \% der überlebenden Patienten drei Monate nach dem Hirnschlag in ihren Aktivitäten des täglichen Lebens wieder unabhängig sind [16].

Die neuronale Plastizität des Gehirns und das hiermit verbundene Rehabilitationspotential zeigen eine ausgeprägte Abhängigkeit vom zeitlichen Verlauf. Der Beginn einer stationären Neurorehabilitation darf nicht ohne Grund verzögert werden. Das Abwarten einer spontanen Teilremission als Voraussetzung für eine multidisziplinäre Rehabilitation würde zu schlechteren Resultaten der anschliessenden Neurorehabilitation führen. Die neuronale Plastizität des Gehirns ist nur bei Beginn der stationären Neurorehabilitation in den ersten Wochen nach dem Hirnschlag therapeutisch optimal zu nutzen, ein verzögerter Beginn (20 bzw. 30 Tage nach dem Hirnschlag) verschlechtert eindeutig das funktionelle Ergebnis [17, 18]. 


\section{Literatur}

1 Indredavik B, Bakke F, Slordahl SA et al. Stroke Unit Treatment. 10-Year Follow-Up. Stroke. 1999; 30:1524-7.

2 Stroke Unit Trialists Collaboration. Organised inpatient (stroke unit) care for stroke. Cochrane Database Syst Rev. 2002;(1): CD000197 (Update of: Cochrane Database Syst Rev. 2000;(2): CD000197). Reprint The Cochrane Library 2006, Issue 4.

3 Langhorne P, Pollock A. What are the components of effective stroke unit care? Age Ageing. 2002; 31(5):365-71

4 Misselwitz B, Puschendorf W, Sünkeler IH. Wie stark ist der Alterseffekt auf den Rehabilitationserfolg nach Schlaganfall? Akt Neurologie. 2005; 32:S126.

5 Reutter-Bernays D, Rentsch HP. Rehabilitation of the elderly patient with stroke: an analysis of short-term and long-term results. Disabil Rehabil. 1993;15:90-5.

6 Nakayama H, Jorgensen HS, Raaschou HO, Olsen TS. The influence of age on stroke outcome. The Copenhagen Stroke Study. Stroke. 1994; 25:808-13.

7 Paolucci S, Antonucci G, Troisi E, et al. Aging and stroke rehabilitation. A case-comparison study. Cerebrovasc Dis. 2003;15:98-105.

8 Ergeletzis D, Kevorkian CG, Rintala D. Rehabilitation of the older stroke patient: functional outcome and comparison with younger patients. Am J Phys Med Rehabil. 2002;81(12):881-9.
9 Lieberman D, Lieberman D. Rehabilitation following stroke in patients aged 85 and above. J Rehabil Res Dev. 2005;42(1):47-53.

10 Bagg S, Pombo AP, Hopman W. Effect of age on functional outcomes after stroke rehabilitation. Stroke. 2002;33:179-85.

11 Luk JK, Cheung RT, Ho SL, Li L. Does age predict outcome in stroke rehabilitation? A study of 878 chinese subjects. Cerebrovasc Dis. 2006;21(4): 229-34.

12 Kugler C, Altenhoner T, Lochner P, Ferbert A. Hessian Stroke Data Bank Study Group ASH. Does age influence early recovery from ischemic stroke? A study from the Hessian Stroke Data Bank. J Neurol. 2003;250:676-81.

13 WHO: www.who.int/disabilities/care/en/.

14 United Nations, Vereinte Nationen: www.un.org/esa/socdev/enable/dissre03.htm.

15 EUROPARAT Ministerkomitee: www.coe.int/t/e/ social_cohesion/soc-sp/Rec(2006)5\%20German .doc.

16 The $2^{\text {nd }}$ Helsingborg Consensus Conference (22 - 24 March 2006). World Neurology, Volume 21, Number 2, Page 16, June 2006.

17 Paolucci S, Antonucci G, Grasso MG, et al. Early versus delayed inpatient stroke rehabilitation: a matched comparison conducted in Italy. Arch Phys Med Rehabil. 2000;61:695-700.

18 Salter K, Jutai J, Hartley M et al. Impact of early vs delayed admission to rehabilitation on functional outcomes in persons with stroke. J Rehabil Med. 2006;38:113-7. 